EGU21-12942, updated on 17 Dec 2021

https://doi.org/10.5194/egusphere-egu21-12942

EGU General Assembly 2021

(c) Author(s) 2021. This work is distributed under

the Creative Commons Attribution 4.0 License.

\title{
Covid-19-related air composition changes over China based on TROPOMI and IASI observations, in situ data and model simulations
}

Trissevgeni Stavrakou ${ }^{1}$, Jean-François Müller ${ }^{1}$, Maite Bauwens ${ }^{1}$, Thierno Doumbia ${ }^{2}$, Nellie Elguindi ${ }^{2}$, Sabine Darras ${ }^{2}$, Claire Granier Claire Granier ${ }^{3,4}$, Yiming Liu Yiming Liu ${ }^{5}$, Xiaoqin Shi ${ }^{6}$, Idir Bouarar $^{6}$, Guy Brasseur ${ }^{6}$, Tao Wang ${ }^{5}$, Henk Eskes ${ }^{7}$, Isabelle De Smedt ${ }^{1}$, Lieven Clarisse ${ }^{8}$, Pierre François Coheur ${ }^{8}$, and Bruno Franco ${ }^{8}$

${ }^{1}$ Royal Belgian Institute for Space Aeronomy, Brussels, Belgium (jenny@oma.be)

${ }^{2}$ Observatoire Midi-Pyrénées, Toulouse, France

${ }^{3}$ Laboratoire d'Aérologie, CNRS, Université de Toulouse, France

${ }^{4} \mathrm{NOAA} / E S R L / C S D \square$ CIRES/University of Colorado, Boulder, CO, USA

${ }^{5}$ Hong Kong Polytechnic University

${ }^{6}$ Max Planck Institute for Meteorology, Hamburg, Germany

${ }^{7}$ Royal Netherlands Meteorological Institute (KNMI), Netherlands

${ }^{8}$ Université libre de Bruxelles (ULB), Spectroscopy, Quantum Chemistry and Atmospheric Remote Sensing (SQUARES),

Brussels, Belgium

The worldwide spread of Covid-19 pandemic caused a dramatic cutback of human activities and triggered a large-scale atmospheric composition experiment. This unfortunate situation provides the opportunity to investigate the response of atmospheric composition to the shutdown measures. Our focus will be on China, where the pandemic emerged in January 2020, and thence strict lockdowns were enforced. Substantial, large-scale decreases in pollutants levels over China and subsequent recovery were revealed by spaceborne observations from TROPOMI instrument on board Sentinel-5 Precursor, as well as by in situ measurements. Most published work on this topic relied on observed changes in column abundances of nitrogen dioxide $\left(\mathrm{NO}_{2}\right)$, a predominantly anthropogenic compound and an important precursor for ozone production and secondary aerosol formation. Our work adds to this picture by studing the evolution of two other satellite-derived compounds, formaldehyde $(\mathrm{HCHO})$ and peroxyacylnitrate (PAN), observed by TROPOMI and IASI, respectively. $\mathrm{HCHO}$ is an intermediate product in the chemical processing of volatile organic compounds (VOCs) of anthropogenic and natural origin. PAN is formed in the oxidation of anthropogenic and biogenic VOCs, and constitute the principal tropospheric $\mathrm{NO}_{x}$ reservoir, enabling the transport and release of $\mathrm{NO}_{x}$ away from the sources. Chemistry-transport simulations of PAN are challenging due to large uncertainties in formation mechanisms and precursor emissions. We will evaluate and analyze the observed variability of $\mathrm{NO}_{2}, \mathrm{HCHO}$, and PAN columns using model simulations with the MAGRITTE v1.1 regional CTM run at $0.5^{\circ} \times 0.5^{\circ}$ resolution over China for 2019 and 2020. The model uses updated anthropogenic emissions from the CONFORM dataset, which takes into account the reductions during the shutdowns based on traffic and other economic activity data. 
